\title{
Silencing Snail suppresses tumor cell proliferation and invasion by reversing epithelial-to-mesenchymal transition and arresting G2/M phase in non-small cell lung cancer
}

\author{
XUEYING YANG* ${ }^{*}$ MENGMENG HAN*, HAIBO HAN, BINGJING WANG, \\ SHENG LI, ZHIQIAN ZHANG and WEI ZHAO \\ Key Laboratory of Carcinogenesis and Translational Research (Ministry of Education), \\ Department of Cell Biology, Peking University Cancer Hospital and Institute, Beijing 100142, P.R. China
}

Received November 10, 2016; Accepted February 9, 2017

DOI: $10.3892 /$ ijo.2017.3888

\begin{abstract}
Epithelial-to-mesenchymal transition (EMT) is essential for tumor invasion and metastasis. Snail has been proven to be a key regulator of EMT. Several studies have shown compelling evidence that Snail is also an important regulator of tumor growth and aggression; however, the role of Snail in the cell cycle has not been clarified. We decreased Snail expression by siRNA transfection and lentiviral-mediated RNAi, to explore the effect of silencing Snail on the tumorigenicity and migration of lung carcinoma (lung cancer) cells. The results showed that silencing Snail conferred significant anti-proliferative activity and inhibited cell migration, tumor growth and metastasis both in vitro and in vivo. To understand the mechanism of these effects, we further investigated correlations among Snail expression, EMT and cell cycle. Significantly, Snail knockdown reversed EMT processes in lung cancer cells. Furthermore, the cyclin-dependent kinase inhibitor P21 was upregulated after silencing Snail. P21 upregulation manifested its tumor suppressor effects and arrested cells in the G2/M phase, not the G1/S phase following Snail depletion in lung cancer cells. These data suggest that silencing Snail decreases the malignant behaviors of lung cancer cells by reversing EMT processes and causing cell cycle defects.
\end{abstract}

Correspondence to: Dr Wei Zhao, Key Laboratory of Carcinogenesis and Translational Research (Ministry of Education), Department of Cell Biology, Peking University Cancer Hospital and Institute, Beijing 100142, P.R. China

E-mail: linelong@126.com

${ }^{*}$ Contributed equally

Abbreviations: NSCLC, non-small cell lung cancer; EMT, epithelialto-mesenchymal transition; CDKs, cyclin-depended kinases; siRNA, small interfering RNA

Key words: Snail, epithelial-to-mesenchymal transition, non-small cell lung cancer, cell cycle

\section{Introduction}

Lung cancer is a major public health problem and the most frequent cause of cancer-related mortality worldwide, with estimated nearly 244,000 new cases and 158,000 deaths annually in the United States (1). In China, there were 733,000 new cases and 610,000 deaths in 2016 (2). In fact, non-small cell lung cancer (NSCLC) is one of the most prevalent histologic types of lung cancer all over the world. Despite considerable advances in early detection and conventional therapies, the 5-year survival rate of patients with NSCLC is $<40 \%$ (3), The primary cause of mortality in NSCLC is widespread metastasis (4). Thus, studying the molecular mechanisms that facilitate tumor invasion and metastasis is of great importance and could reveal new therapeutic measures for treating lung cancer, leading to improved patient survival rates.

Epithelial-to-mesenchymal transition (EMT) is a cellular process that allows cells to lose their epithelial characteristics and adopt a mesenchymal phenotype and mesenchymal characteristics to increase motility and invasion (5). Recent studies have shown that EMT occurs at the invasive front of tumors and is a critical event for cancer metastasis (6). Through EMT, tumor cells attain invasive properties and have the ability to enter the surrounding stroma, leading to the creation of a favorable microenvironment for metastasis (7), which is characterized by the suppression of the adhesion protein E-cadherin (8). Thus, the cadherin family contributes to cancer progression and metastasis. Snail, the SNAI1 gene product, is a member of the Snail family of zinc-finger transcription factors and triggers EMT by repressing E-cadherin expression, ultimately enhancing cancer invasion in several malignancies such as breast, ovarian, skin, hepatocellular and head and neck carcinomas $(9,10)$. In many cancers, Snail expression is not only associated with cancer invasion, but also highly correlated with lymph node and distant metastasis through E-cadherin repression $(11,12)$. Furthermore, Snail can also function as a negative regulator of cell growth by repressing cyclin-dependent kinases (CDKs) to block the cell cycle, which is also involved in tumor development and malignancy $(13,14)$. 
Our previous study indicated that the lung cancer cell lines A549 and PG have higher Snail mRNA levels than those with a normal epithelial phenotype [e.g., human fetal lung fibroblasts (HLF) and a spontaneously immortalized human fetal lung epithelial cell line (HLEC)]. As a transcriptional activator, Snail binds E-boxes in the myosin Va promoter, promoting tumor migration and metastasis (15). In this study, we hypothesized that Snail could affect cell proliferation, altering the progression and invasion of lung cancer in vitro and in vivo. Additionally, to explore how Snail is correlated with EMT and cell cycle progression in lung cancer cells, we tested phenotype, cadherin expression and cell cycle check point gene expression in our models. Interestingly, the cell cycle regulator P21 was upregulated following Snail knockdown by small interfering RNA (siRNA), and was correlated to decreased PCNA expression. Recently reported as genes induced by tricostatin A, P21 and P27 as well as Snail and Slug were upregulated in normal epithelial cells (16). To clearly demonstrate which is driving these phenotypes, we confirmed that P21 was directly upregulated by Snail knockdown and suppressed the proliferation of lung cancer cells by arresting at the G2/M phase of the cell cycle through inhibition of Cdc2 (CDK1). These results suggest that the loss of Snail decreased lung cancer proliferation and invasion via contributions of EMT and cell cycle.

\section{Materials and methods}

Cell cultures. The human lung carcinoma cell lines A549 (American Type Culture Collection) and PG (established from a lung giant cell carcinoma by Dr Bing-Quan Wu, Department of Pathology, Peking Medical University) were cultivated and maintained in RPMI-1640 medium (Invitrogen, Grand Island, $\mathrm{NY}$, USA) under $95 \%$ humidified $5 \% \mathrm{CO}_{2}$ at $37^{\circ} \mathrm{C}$ incubator. Medium contains $10 \%$ fetal bovine serum (FBS), $100 \mathrm{U} / \mathrm{ml}$ penicillin and $100 \mathrm{~g} / \mathrm{ml}$ streptomycin.

Snail interfering RNA transfection. The oligonucleotides for human Snail small interfering RNAs (siRNAs) and control were synthesized by Invitrogen. The 19-bp sequences of Snail siRNA were designed by OligoEngine Workstation software: 5'-CATCCGAAGCCACACGCTG-3' (siRNA1); 5'-GCT GGCAGCCATCCCACCT-3' (siRNA2); 5'-GCACAACAA GCCGAATACA-3' (Scramble). A549 cells were transfected with 200 pmol each siRNAs by Lipofectamine 2000 (Invitrogen) according to the manufacturer's instructions. Blank vector was transfected into controls. At $72 \mathrm{~h}$ after transfection, the total RNA was extracted and RT-PCR was performed to assess mRNA levels of Snail, in order to confirm which siRNA was more effective.

Subsequently, $2 \mu \mathrm{g}$ of pSuper-Snail siRNA2 was constructed into A549 cells in 35-mm diameter culture dish. Blank vector were transfected into control dishes. After $24 \mathrm{~h}$ post-transfection, $400 \mu \mathrm{g} / \mathrm{ml} \mathrm{G} 418$ was added to the culture medium for 12 days and 15 G418-resistant cell clones were picked. Four clones named C1-C4 were selected from the 15 to analyze the mRNA and protein levels of Snail by RT-PCR and western blot analysis.

Lentivirus packaging and infection. Plenti4-Snail siRNA2 plasmid was constructed by inserting the Snail siRNA2 fragment into a plenti4 expression vector and was verified by DNA sequencing. The mixed plasmids (3 $\mu \mathrm{g}$ of plenti4-control or plenti4-Snail siRNA2 and $9 \mu \mathrm{g}$ of lentivirus packaging vectors) were transfected into the $293 \mathrm{FT}$ packaging cells using Lipofectamine 2000. Virus-containing supernatants were harvested $48 \mathrm{~h}$ later. The supernatant was then filtered and added to A549 and PG cells. Virus-infected cells were selected by Zeocin $(200 \mu \mathrm{g} / \mathrm{ml}) 24 \mathrm{~h}$ after infection. The Zeocin-resistant clones were pooled and maintained in regular RPMI-1640 media with 10\% FBS containing Zeocin $(100 \mu \mathrm{g} / \mathrm{ml})$. Established stable cell lines were used in subsequent experiments.

Reverse transcription-polymerase chain reaction. Total cellular RNA was extracted using TRIzol reagent (Invitrogen, Carlsbad, CA, USA) according to the manufacturer's protocol. For semi-quantitative reverse transcription-polymerase chain reaction (RT-PCR), the cDNAs were synthesized from $2 \mu \mathrm{g}$ of total RNA in a total volume of $20 \mu \mathrm{l}$ using the Superscript III kit (Invitrogen). The cDNA as the template were subjected to PCR reaction. The housekeeping gene GAPDH was used as an internal control. The final PCR products were visualized in agarose gel electrophoresis. The PCR primers of each gene are described in Table I.

Western blot analysis. The cells were washed twice with cold PBS and harvested with a scraper. Cells were centrifuged at $3000 \mathrm{~g}$ for $30 \mathrm{sec}$. The pellets were lysed in RIPA buffer (50 mM Tris, pH 7.4, $150 \mathrm{mM} \mathrm{NaCl,} 1 \%$ Triton X-100, $1 \%$ sodium deoxycholate, $0.1 \%$ SDS, 2 mM EDTA, $1 \mathrm{mM}$ sodium orthovanadate, $1 \mathrm{mM}$ phenylmethylsulfonyl fluoride and $5 \mu \mathrm{g} / \mathrm{ml}$ leupeptin) containing complete protease inhibitor cocktail (Roche, Mannheim, Germany). Equal amounts of protein lysates were resolved by SDS-PAGE, and then were transferred onto polyvinylidene difluoride (PVDF) membrane (Millipore, Bedford, MA, USA). Non-specific binding sites on the membranes were blocked in Tris-buffered saline (TBS, $50 \mathrm{mM}$ Tris- $\mathrm{HCl} \mathrm{pH} \mathrm{7.5,150} \mathrm{mM} \mathrm{NaCl}$ ) containing 5\% fat-free dried milk and incubated for $1 \mathrm{~h}$ at room temperature. After immunoblotting with primary antibodies at $4^{\circ} \mathrm{C}$ overnight, the membrane was washed 3 times with $0.05 \%$ Tween-20 in TBS. Subsequently, the membranes were incubated with secondary antibody for $1 \mathrm{~h}$ at $37^{\circ} \mathrm{C}$. Detection of the reactive protein performed was detected using Immobilon ${ }^{\mathrm{TM}}$ Western Chemiluminescent HRP substrate (Millipore). The primary antibodies used are shown in Table II. The HRP-conjugated antibodies were used as secondary antibody (1:50,000 dilute, Jackson ImmunoResearch, West Grove, PA, USA).

cDNA microarray. Equal amounts of cDNA from A549 cultured in stable Snail silence cell and control cells were labeled with $\mathrm{Cy} 3$ and $\mathrm{Cy} 5$, respectively, and then hybridization was carried out according to the manufacture's protocol using human genome cDNA microarray consisting of 14,592 genes (Shanghai BioChip Co., Ltd., Shanghai, China). Genes were considered to be up or downregulated when the fluorescent intensity ratio between $\mathrm{Cy} 3$ and $\mathrm{Cy} 5$ cells was $>2$ or $<0.5$. The experiment was repeated once. Hierarchical clustering of regulated genes in this study was measured by Genespring software. 
Table I. PCR primer sequences.

qRT-PCR primers

\begin{tabular}{lllc}
\cline { 2 - 4 } Gene name & \multicolumn{1}{c}{ Sense } & \multicolumn{1}{c}{ Antisense } & Size (bp) \\
\hline SNAII & 5'-CTGCAGGACTCTAATCCAG-3' & 5'-CAAGGAAGAGACTGAAGTAG-3'; & 300 \\
SNAI2 & 5'-TCGGACCCACACATTACCTT-3' & 5'-TGACCTGTCTGCAAATGCTC-3' & 145 \\
TWIST1 & 5'-CCTTCTCGGTCTGGAGGAT-3' & 5'-TCCATTTTCTCCTTCTCTGGAA-3' & 127 \\
CDH1 & 5'-TCCATTTCTTGGTCTACGCCT-3' & 5'-TCACCTTCAGCCATCCTGTTT-3' & 363 \\
CDH2 & 5'-GTGCCATTAGCCAAGGGAATT-3' & 5'-GGAGGAATTCCATTGTCAGAAG-3' & 350 \\
CD $N$ N1A & 5'-AGTCAGTTCCTTGTGGAGCC-3' & 5'-CATGGGTTCTGACGGACAT-3' & 108 \\
GAPDH & 5'-GACCCCTTCATTGACCTCAAC-3' & 5'-CTTCTCCATGGTGGTGAAGA-3' & 219
\end{tabular}

Table II. Antibodies used in western blots and immunofluorescence analysis.

\begin{tabular}{lllll}
\hline Name & Vender & Cat no. & \multicolumn{1}{c}{ Species } & Dilution \\
\hline Snail & R\&D & 965619 & Goat polyclonal IgG & $1: 2,000$ \\
E-cadherin & Santa Cruz & sc52328 & Mouse monoclonal IgG & $1: 2,000$ \\
N-cadherin & Sigma & C2342 & Mouse monoclonal IgG & $1: 2,000$ \\
PCNA & Santa Cruz & sc56 & Mouse monoclonal IgG & $1: 2,000$ \\
P21 & Santa Cruz & sc397 & Mouse monoclonal IgG & $1: 2,000$ \\
CDK1 & Abgent & Ap1497a & Rabbit polyclonal IgG & $1: 1,000$ \\
CDK2 & Santa Cruz & sc163 & Rabbit polyclonal IgG & $1: 2,000$ \\
CDK4 & Abgent & Ap20515b & Rabbit polyclonal IgG & $1: 2,000$ \\
Actin & Roche & 1378996 & Mouse monoclonal IgG & $1: 10,000$ \\
\hline
\end{tabular}

Cell proliferation assay. To compare proliferation rates in vitro, cells were seeded at a density of $1 \times 10^{4}$ cells/well in 24-well culture plates with $400 \mu \mathrm{l} /$ well of growth medium. Each day (5 days), cells from duplicates were collected after trypsinization. Cells were counted with a hemocytometer under a microscope. Cell Generation Time $(\mathrm{GT}=\mathrm{Tn} / 5 ; \mathrm{Tn}=\mathrm{t} /$ $\ln (\mathrm{Nf} / \mathrm{Ni}) / \ln 2 ; \mathrm{Nf}$, final cell number at time $\mathrm{t}$; $\mathrm{Ni}$, initial plated cell number; ln, natural logarithm) was calculated by plotting the graph of cell counting versus time.

Wound healing assay. The cells were seeded in 6-well dishes with proper concentration $\left(\sim 3 \times 10^{5}\right.$ per well). Confluent cells were scratched $24 \mathrm{~h}$ later by a disinfected Eppendorf Tip. The detached cells were washed away with PBS and fresh medium was added. Cultures were incubated at $37^{\circ} \mathrm{C}$ with $5 \% \mathrm{CO}_{2}$ for a further $24 \mathrm{~h}$. The images of the wound width were captured by an inverted microscope (Leica, Wetzlar, Germany) at 0 and $24 \mathrm{~h}$ post-scratch, respectively. Distance of cell movement was measured by LAF software (Leica).

Boyden chamber assay. The cell migration and invasion capacity was analyzed by using Boyden chamber assay. Briefly, a total of $5 \times 10^{4}$ cells were re-suspended in $200 \mu \mathrm{l}$ serum-free RPMI-1640 media and plated in the upper chambers of Transwell (24-well insert; pore size, $8 \mu \mathrm{m}$; Corning). The lower chambers were filled with $800 \mu$ l RPMI-1640 containing $10 \%$ FBS. After cultures were incubated for $24 \mathrm{~h}$ at $37^{\circ} \mathrm{C}$, non-invasive cells were wiped off from the upper surface and the invasive cells that were attached on the lower surface of the chamber were stained with $0.5 \%$ crystal violet, followed by fixed with methanol. The average number of cells was determined by counting five random fields in triplicates by light microscopy.

Cell cycle analysis. Cells $\left(5 \times 10^{5}\right.$ cells/well) were plated in 6-well plates and incubated for $24 \mathrm{~h}$. Then, the cells were collected by trypsinization, washed with PBS twice, and fixed with $70 \%$ pre-chilled ethanol at $4^{\circ} \mathrm{C}$ overnight. The following day, the cells were pelleted, treated with $50 \mu \mathrm{g} / \mathrm{ml}$ of RNase A (Roche) for $30 \mathrm{~min}$ at room temperature followed by propidium iodide (Invitrogen) staining at room temperature for $30 \mathrm{~min}$. Finally, the stained cells were examined by flow cytometry (BD, San Jose, CA, USA). The percentage of cell population in different phases of cell cycle was analyzed using CFlow software V1.0.

Immunofluorescence staining. Cells growing on coverslips were fixed with $4 \%$ formaldehyde for $3 \mathrm{~min}$ at room temperature, followed by washing with $0.5 \%$ Triton $\mathrm{X}-100$ in PBS three times. Primary antibodies, e.g., $\mathrm{N}$-cadherin and E-cadherin (described above), were next incubated for $1 \mathrm{~h}$ at room temperature. After being washed with $0.5 \%$ Triton X-100 in PBS, the cells were labeled for $1 \mathrm{~h}$ with TRITC-conjugated anti-mouse IgG (1:100, Jackson) or FITC-conjugated antirabbit $\operatorname{IgG}$ (1:100, Jackson) secondary antibody solution at room temperature and washed again. Afterwards, the cell nuclei were stained by DAPI (Sigma-Aldrich, St. Louis, MO, 
A

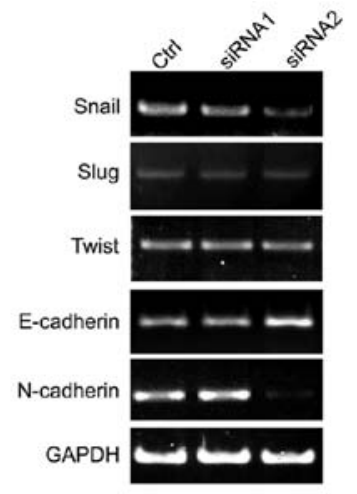

C

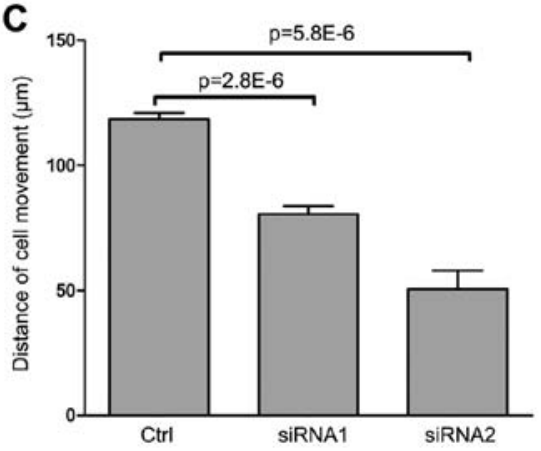

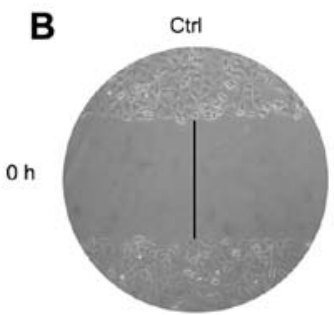
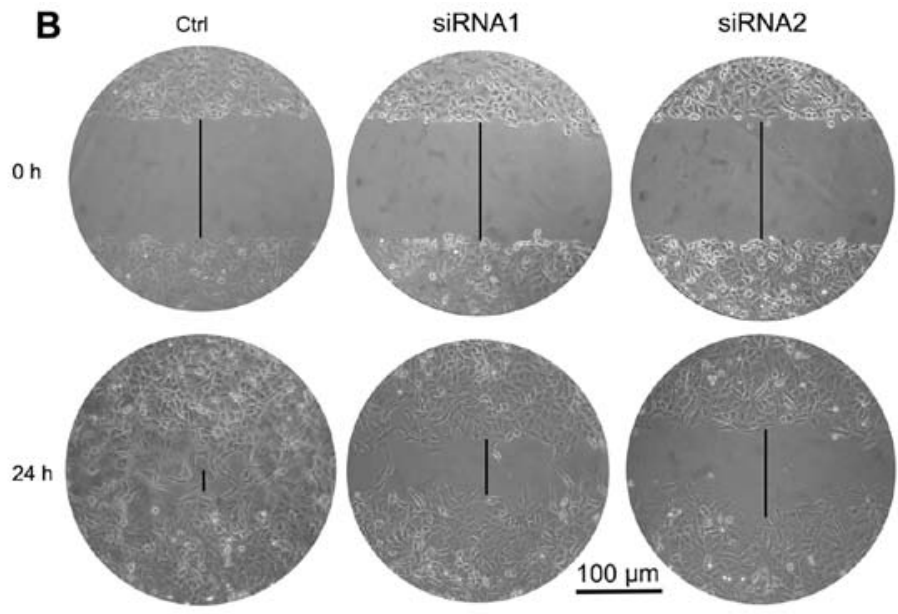

D

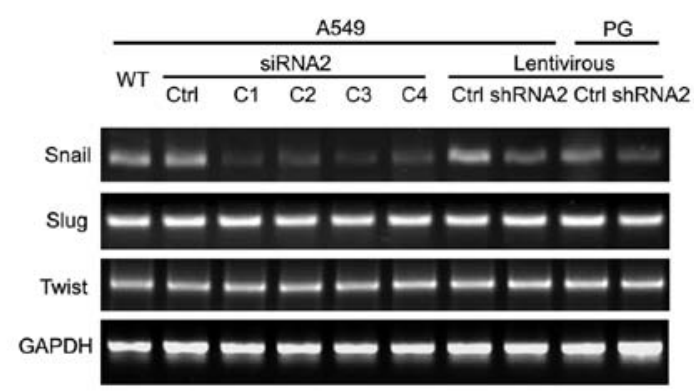

Figure 1. Knockdown of Snail by RNA interference in lung cancer cells. The indicated gene expression of Snail siRNAs (1 and 2) and scramble control were examined at the mRNA levels by semi-quantitative RT-PCR in the A549 cells. The GAPDH expression served as an internal control (A). Comparison of migration capability in A549 cells transiently transfected with Snail siRNA (1 and 2) and the control cells assessed by wound healing assay. Representative images of wound closure at 0 and $24 \mathrm{~h}$ post-injury are presented (B). The column diagram shows the average distance of cell movement at $24 \mathrm{~h}$ post-injury with its associated standard deviation (C). Semi-quantitative RT-PCR was used to examine the mRNA expression levels of Snail, Slug and Twist among the pSuper-Snail siRNA2 clones (C1-C4), the plenti4-Snail shRNA2 lentivirus-infected A549 and shRNA2 lentivirus-infected PG cells (D). Bars, $100 \mu \mathrm{m}$.

USA) in PBS for 3 min. Staining was detected on a Leica SP5 laser confocal microscope.

Animal experiments. For evaluating the tumorigenicity in animals, cells were trypsinized, centrifuged, and re-suspended in PBS. Suspensions containing $2 \times 10^{5}$ cells in $0.2 \mathrm{ml}$ PBS were implanted subcutaneously into the under arm area of athymic nude mice (BALB/c, Nu/Nu; 4-6-week-old, Vitalriver Laboratory Animals, Beijing, China) (5 mice/group). Tumor formation was examined two times each week by calipers and tumor volumes were estimated as follows: $\mathrm{V}=$ the length $\mathrm{x}$ (the width 2)/2. After 26 days, all the mice were euthanized and the tumors were excised and weighed.

The detection of PG cells metastasis in mouse lung was performed as descripted (17). Briefly, genomic DNA was extracted from fresh mouse lung tissues. Quantitative real-time PCR was used to detect human Alu sequences by using primers (sense, 5'-ACG CCT GTA ATC CCA GCA CTT-3'; and antisense, 5'-TCG CCC AGG CTG GAG TGC A-3') (18). The mouse housekeeping gene GAPDH was used as an internal control. The level of human Alu-sequence was normalized to amount of mouse GAPDH genomic DNA sequence amplified. All animal studies were performed in accordance with the policies of the Institutional Animal Care and Use Committee and were approved by the Institutional Review Board of Peking University School of Oncology.
Statistical analysis. Each experiment was conducted in triplicate. All results are presented as means \pm SD. The comparison between two groups was calculated by Student's t-test with two-tails. $\mathrm{p}<0.05$ was regarded as statistically significant.

\section{Results}

Snail siRNA knockdown in lung cancer cells. We previously demonstrated that Snail mRNA expression was increased in the highly malignant lung cancer cell lines A549, PG and Calu6 compared with the less malignant cell line Calu3 (15). This suggested that Snail might be involved in cancer cell migration. To further explore how Snail expression influences malignant behavior through loss-of-function analysis, we inhibited Snail expression in A549 cells using two sets of siRNAs targeting Snail: siRNA1 and siRNA2. After $48 \mathrm{~h}$ of growth, scramble or Snail siRNAs were transfected into A549 cells. PCR analysis was performed to analyze the Snail and GAPDH mRNA levels. The results showed that both Snail-targeting siRNAs effectively repressed Snail mRNA levels. Moreover, Slug and Twist, two close homologs of Snail, showed no significant changes between the siRNA- or scramble-transfected cells (Fig. 1A). These results showed that siRNA2 was more effective, although both siRNAs were specific for Snail. At the functional level, wound healing assays were performed to assess how these siRNAs suppressed the 
A

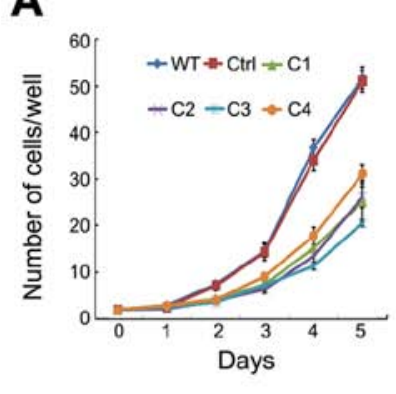

E
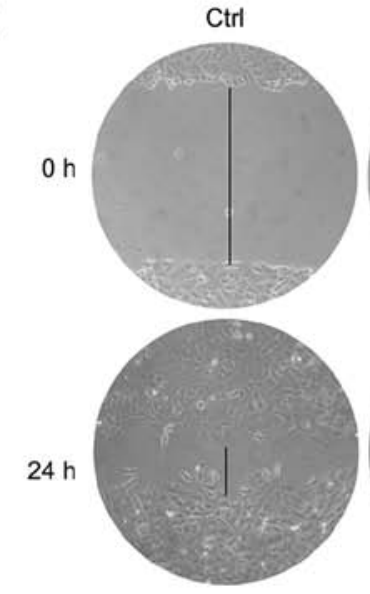

F

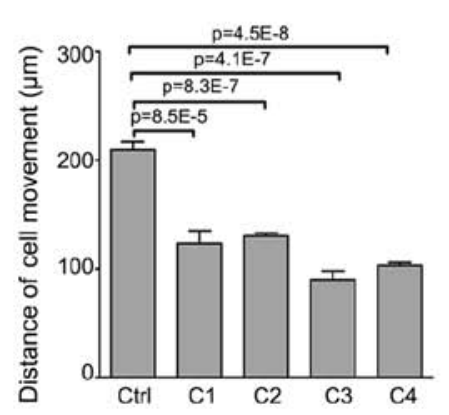

B

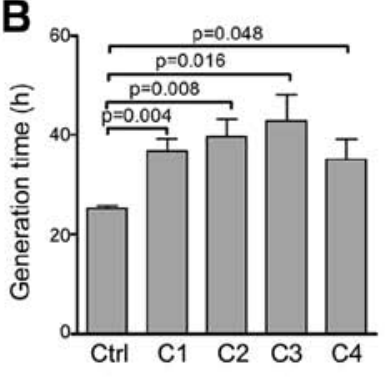

C

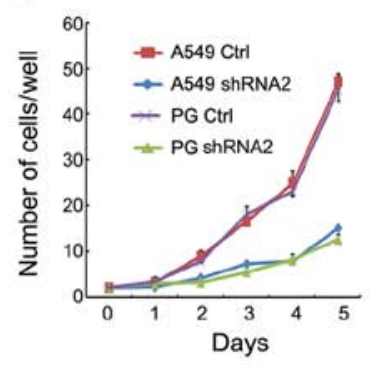

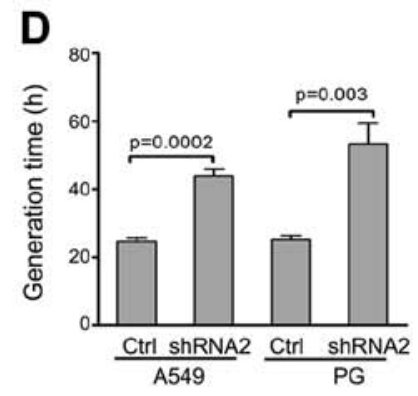

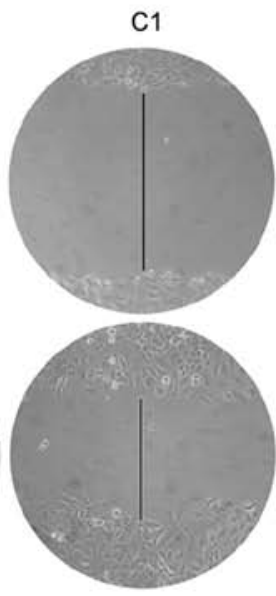

G

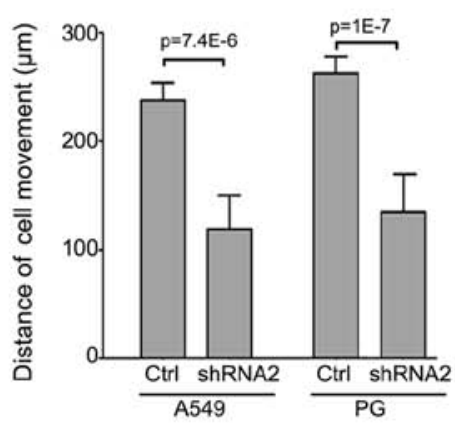

C3
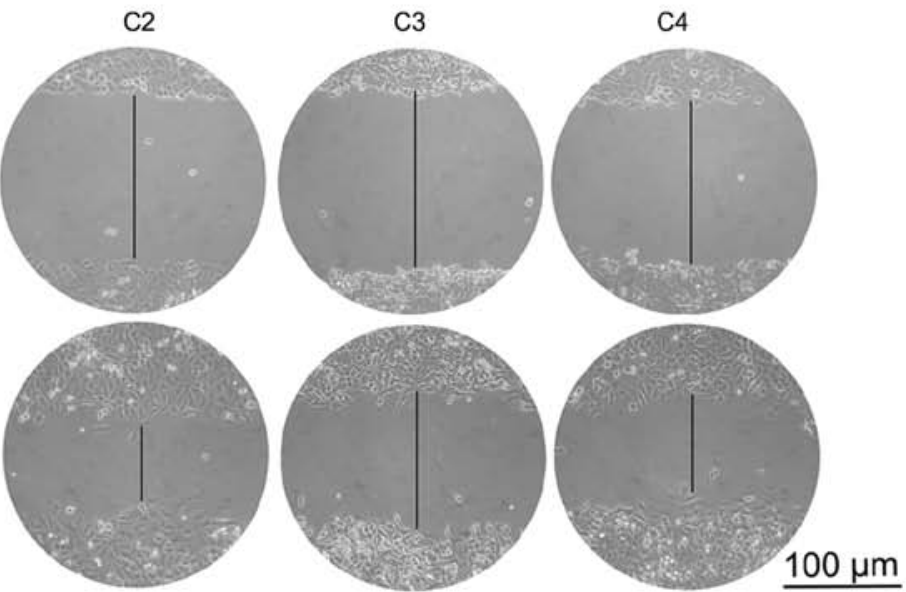

H

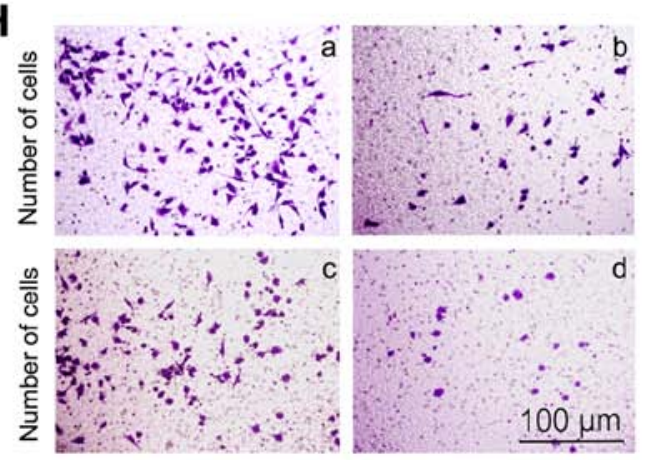

Figure 2. Snail downregulates proliferation and migration behavior of lung cancer cells in vitro. Proliferation of stable Snail siRNA2 transfected clones (A). Bar graphs showed the average generation time of the 4 cell clones (C1-C4) (B). Proliferating stable Snail shRNA2 lentivirus-infected A549 and PG cells (C). Bar graphs showed the average generation time of A549 Snail shRNA2 and PG Snail shRNA2 cells (D). Wound healing assay for cell invasion of the 4 cell clones (C1-C4). Representative images of the wound areas were taken by phase-contrast microscopy at 0 and $24 \mathrm{~h}$ post-scratching (E). The graph displayed the average distance of cell movement at $24 \mathrm{~h}$ post-injury in A549 siRNA clones (F) and in A549 and PG by shRNA2 (G). Representative imaged cells evaluated

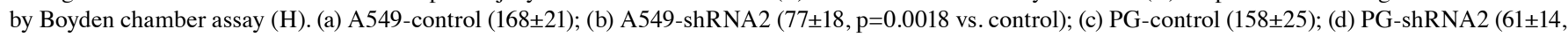
$\mathrm{p}=0.0003$ vs. control). Original magnification, $\mathrm{x} 100$. The data represent the mean \pm SD of triplicate samples. Bars, $100 \mu \mathrm{m}$.

migratory behavior of A549 cells. siRNA2 consistently showed a more significant effect on migration $(50.5 \pm 18.2 \mu \mathrm{m} /$ day $)$, compared with siRNA1 $(80.5 \pm 7.8 \mu \mathrm{m} /$ day $)$ or the scramble control (118.4 $\pm 6.1 \mu \mathrm{m} /$ day) (Fig. 1B and C). Hence, siRNA2 was selected for further experiments.

To generate stable Snail-silenced cells, lentiviruses containing Snail-targeting constructs were used to block Snail expression in A549 and PG cells. Four clones (C1-C4) of Snailsilenced A549 and PG cells were isolated and screened for Snail expression by RT-PCR. Compared with controls, Snail expression was decreased in all four clones, but the interfering efficiency of clone C3 was most dramatic. Similar result was shown in Fig. 1D, the efficiency of Snail shRNA knockdown was obtained by using the lentiviral system.
Behavior of Snail-silenced lung cancer cells. To clearly understand the functional changes associated with in vitro Snail knockdown by RNA interference, we first measured cell growth in the stable Snail-silenced cell lines by cell counting. Snail downregulation significantly reduced growth in A549 clones (Fig. 2A), and the GT averages are shown in Fig. 2B. Similarly, A549 and PG cells infected with Snail-knockdown lentivirus were analyzed for cell growth and GT (Fig 2C and D). The data clearly shows that silencing Snail decreased proliferation of both A549 and PG cells in vitro. Second, to analyze the impact of Snail suppression on cell motility, we performed wounding healing assays to test the migratory ability of each stable cell line. The data showed that clone C3 had a highly decreased migration capacity among the 
A

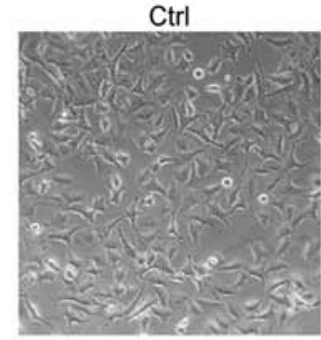

B
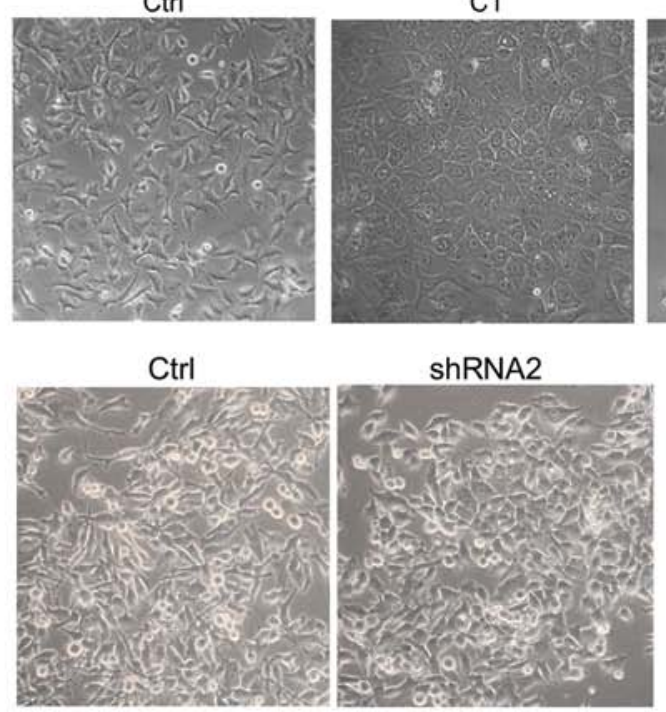

shRNA2

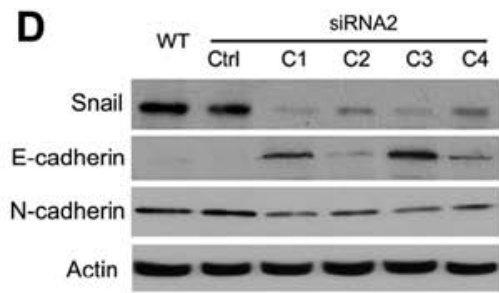

E

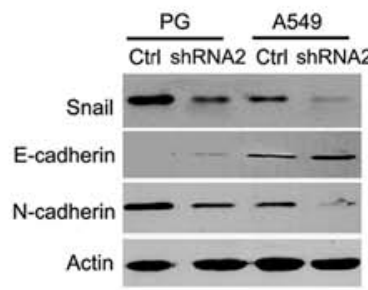

$\mathbf{F}$
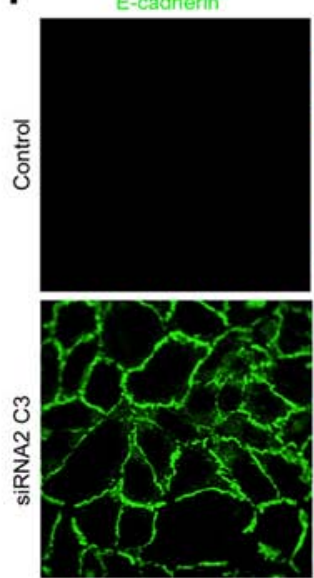

$\mathrm{C} 2$

C3

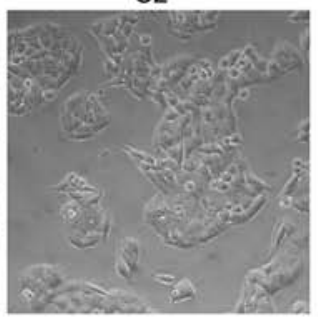

C
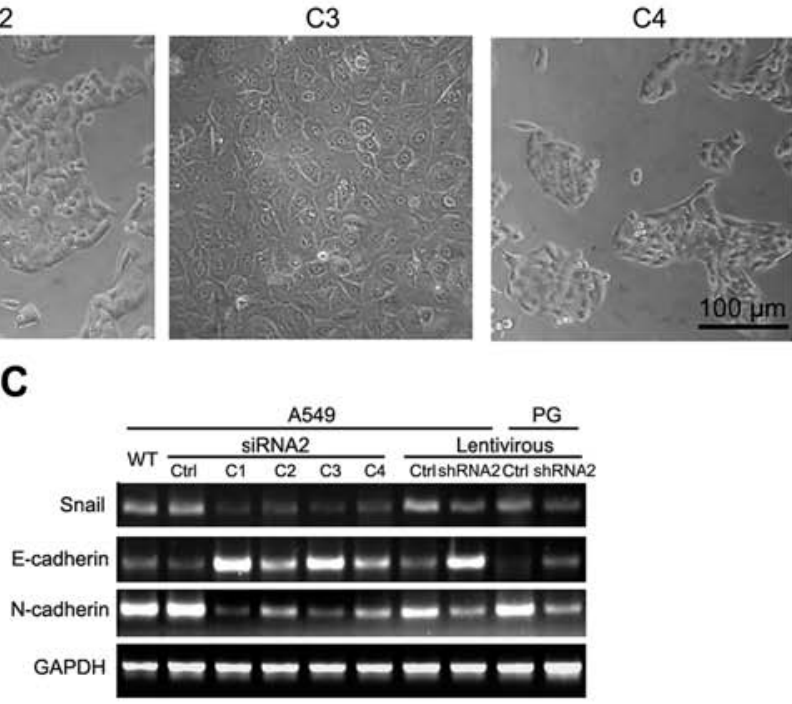

E-cadherin/DAPI
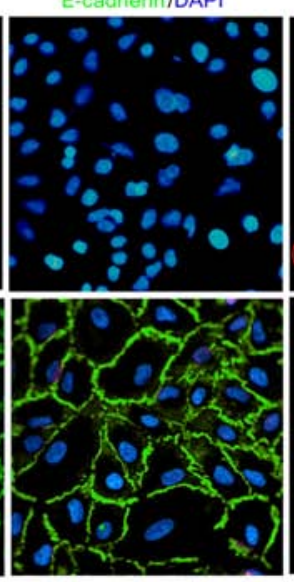

$\mathrm{N}$-cadherin
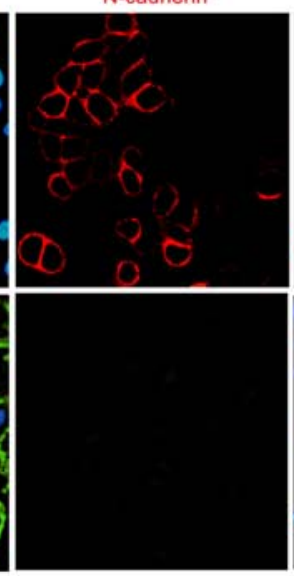

$\mathrm{N}$-cadherin/DAPI

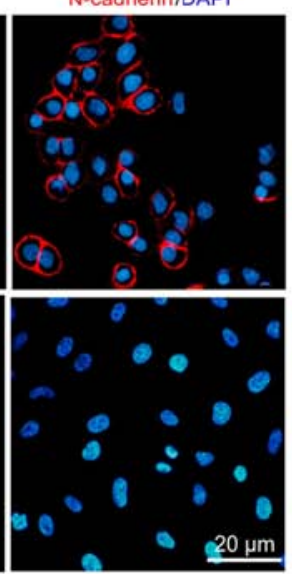

Figure 3. Knockdown of Snail induces reversion of EMT in lung cancer cells. The cellular morphology of the 4 cell clones (C1-C4) and the control were captured by phase-contrast microscopy (A). The morphology of PG Snail siRNA2 stable cells (B). Semi-quantitative RT-PCR was performed to determine the expression of Snail, E-cadherin and N-cadherin mRNA in A549 and PG cells with Snail knockdown (C). Western blot analysis was performed to analyze the expression of Snail, E-cadherin and N-cadherin protein in both cell clones (D) and A549 Snail shRNA2 and PG Snail shRNA2 cells (E). Immunofluorescence assays for EMT markers E-cadherin and N-cadherin expression in clone $\mathrm{C} 3$ and control cells. The nucleus was detected with DAPI (F). Bars, $100 \mu \mathrm{m}$.

four clones and demonstrated the weakest movement ability (Fig. 2E and F). Furthermore, the same suppression of cell motility was demonstrated in A549 and PG cells following Snail knockdown by lentiviral system (Fig. 2G). Third, we analyzed the invasive properties following Snail knockdown using the Boyden chamber assay. As shown in Fig. $2 \mathrm{H}$, the Snail knockdown A549 and PG cells were significantly less invasive than control, A549 invasion was decreased from $168 \pm 21$ to $77 \pm 18$ per well, and PG was decreased from $158 \pm 25$ to $61 \pm 14$ per well. These results indicate that Snail silencing inhibited not only proliferation, but also suppressed the migratory behavior of lung cancer cells in vitro.

Snail downregulation induces a reversion of EMT in lung cancer cells. In a previous study, we performed a series of experiments exploring the phenotypes of Snail knockdown cells, assessing how Snail knockdown suppresses cell proliferation and migration (15). To gain further insight into possible mechanisms underlying Snail knockdown, we examined the morphology of A549 and PG cells following Snail silencing. Of the four A549 clones, C1 and C3 (Fig. 3A) and PG Snail siRNA2 stable cells (Fig. 3B) showed a sheet-like epithelial phenotype with well-organized cell-cell adhesion, whereas control cells had a spindle-shaped mesenchymal morphology. Therefore, we evaluated E-cadherin and $\mathrm{N}$-cadherin expression, two important cadherin family members in cancer biology. A549 and PG cells showed increased E-cadherin and decreased $\mathrm{N}$-cadherin expression at both the mRNA and the protein levels following Snail knockdown (Fig. 3C-E). Among the four clones, C3 had the most significant effect on the expression of EMT-associated markers. To confirm the hypothesis of EMT reversion following Snail knockdown, we assessed the expression and localization of epithelial and mesenchymal markers in clone $\mathrm{C} 3$ using indirect immunofluorescence staining (Fig. 3F). As expected, an increase in E-cadherin expression was observed in clone C3. Conversely, $\mathrm{N}$-cadherin expression was absent with Snail knockdown. In addition, compared with the control, clone C3 had a clear 


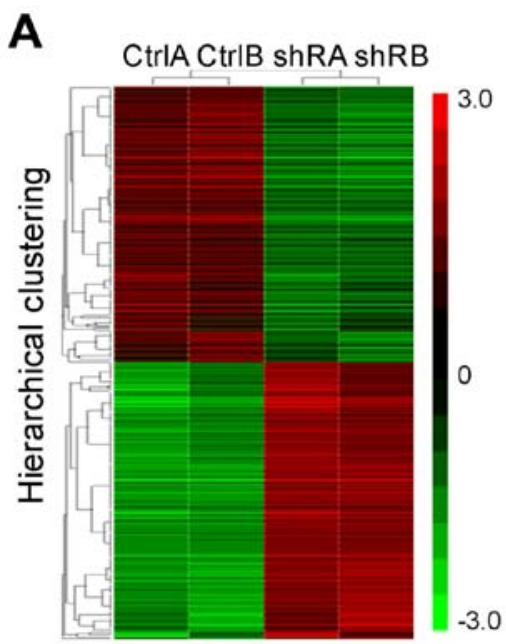

B

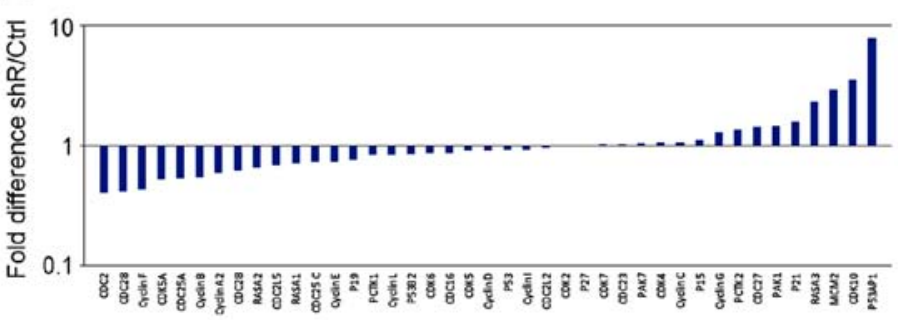

C

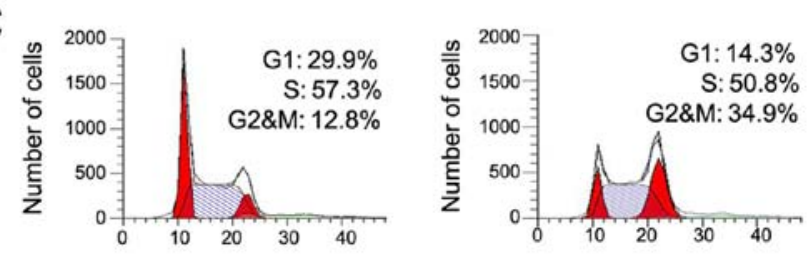

D
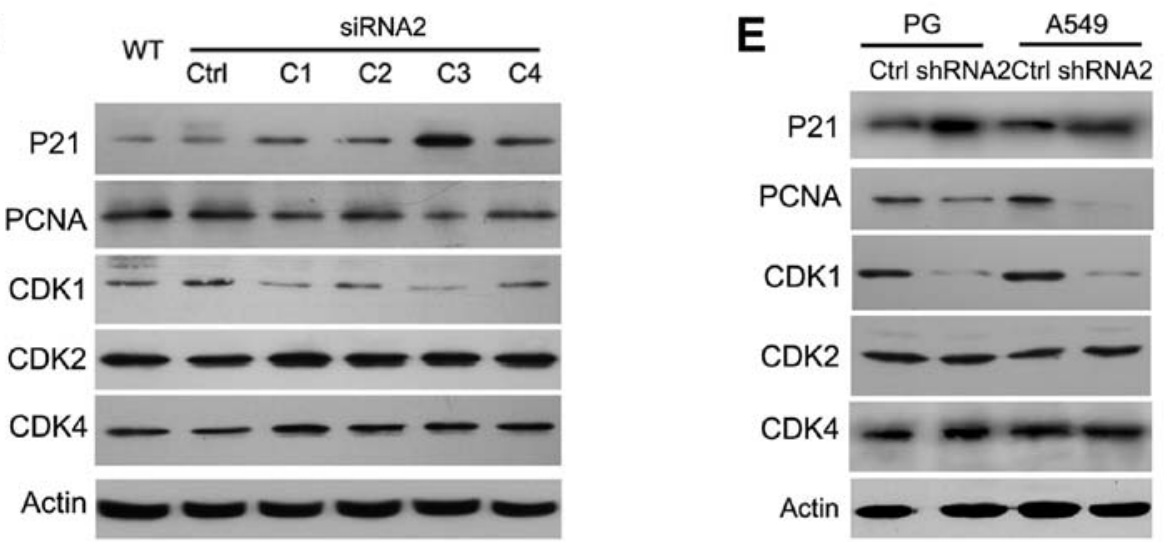

Figure 4. Snail interference arrests in G2/M phase of lung cancer cells. Hierarchical clusters and RNA ratios of differentially expressed genes of A549 control (Ctrl) and Snail shRNA2 (shR) cell cultures (A). Red indicates genes expressed above average; green indicates genes expressed below average and black indicates average expression after significance analysis of microarrays (SAM). (A) 1st data; (B) 2nd data. Difference in gene expression was highly related to cell cycle on gene chips and are listed low to high from left to right (B). Cell cycle of A549 control (left) and Snail shRNA2 (right) were detected by FCS (C). Western blot analysis was performed to analyze the expression of P21, PCNA, CDK1, CDK2 and CDK4 protein in both cell clones (D) and A549 Snail shRNA2 and PG Snail shRNA2 cells (E).

A
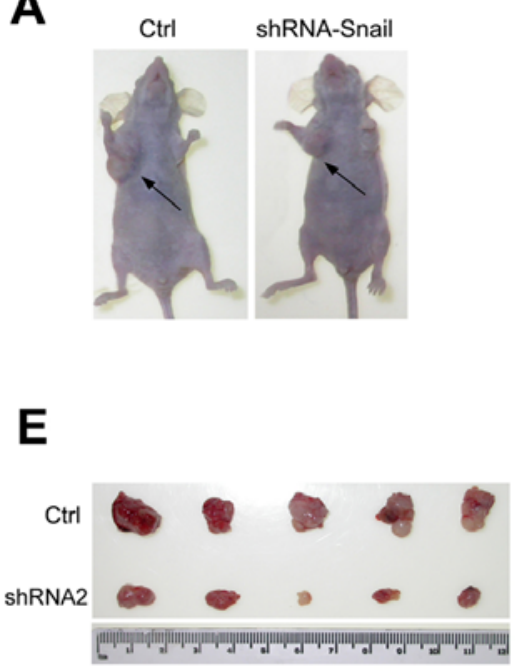
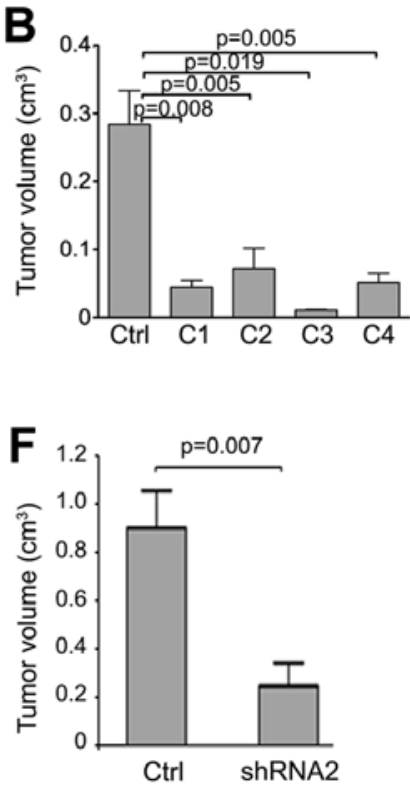

C

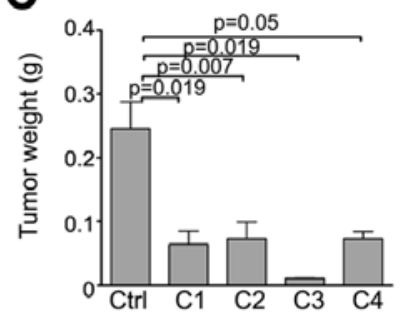

D
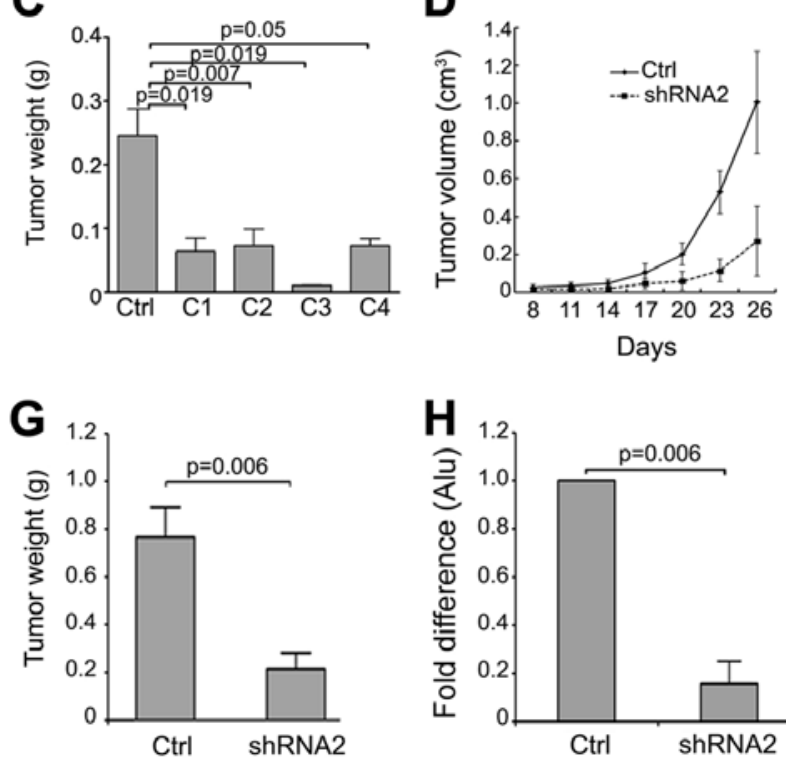

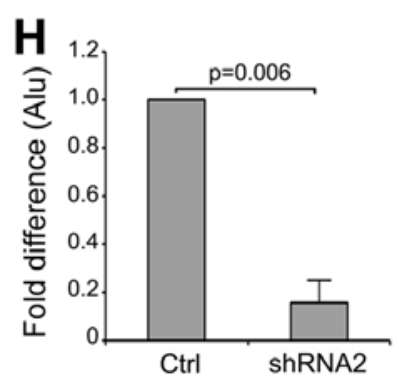

Figure 5. Snail knockdown controls tumorigenicity and metastasis in vivo. The 4 cell clones (C1-C4) and the control were injected into the underarm of mice. Mice were sacrificed and tumors (arrow) were extracted (A). Tumor dimensions and weight were measured. The average tumor volume (B) and weight (C) are shown from the 5 tumors formed on mice by each cell line. The PG Snail shRNA2 and control cells were injected into mice. The curve shows the tumor growth rate of PG Snail shRNA2 cells in nude mice (D). The dissected tumors (E) and the graph show the average tumor volumes (F) and weight $(\mathrm{G})$ from the 5 tumors formed on mice by each group. Relative Alu-sequence expression in mouse lung was quantified by real-time PCR of (H). 
localization of E-cadherin at the cell membrane. These results suggest that Snail silencing affects lung cancer cell migration and metastasis through the reversion of EMT.

A G2/M cell cycle arrest following Snail knockdown. To further investigate mechanisms underlying the suppressed tumorigenicity of Snail-silenced cells, we analyzed data from cDNA microarray analyses to find global gene expression changes associated with Snail knockdown (Fig. 4A). This unbiased approach allowed us not only to find EMT-associated genes, but also genes highly associated with the cell cycle. Associated cell cycle genes were listed from low to high expression responded on mRNA levels including $C d c 2$ (0.4-fold), $C D K 2$ (1.01-fold), $C D K 4$ (1.05-fold), CDK6 (0.86-fold), $P 21$ (1.6-fold) and their related genes (Fig. 4B). Therefore, we next performed cell cycle analysis in A549 cells by flow cytometry. The results showed that the G1 and S periods were down and the G2/M population was especially overrepresented, $\sim 3$-fold higher following Snail knockdown (Fig. 4C). To investigate why the cell cycle was changed, we analyzed the expression of cell cycle-related proteins, such as P21, PCNA, Cdc2, CDK2, and CDK4 by western blot analysis. Our data showed that Snail knockdown led to the upregulation of $\mathrm{P} 21$ and downregulation of $\mathrm{Cdc} 2$, but did not affect CDK 2 or CDK4 expression (Fig. 4D and E). These data indicated that Snail knockdown may inhibit cell growth via causing a $\mathrm{G} 2 / \mathrm{M}$ cell cycle arrest.

Snail knockdown inhibits tumorigenicity and metastasis of lung cancer cells in vivo. We next investigated how Snail knockdown modulates tumorigenicity and metastasis of lung cancer cells in vivo, utilizing the nude mouse xenograft model (Fig. 5A). A decrease in tumor size and weight were observed in tumors formed from the four Snail knockdown A549 clones compared to those from control cells (Fig. 5B and C). C3 tumors tended to be the smallest among all clones. Moreover, the plotted growth curves of tumor formation were markedly different between PG Snail shRNA2 cells and control cells (Fig. 5D). Compared to the controls, PG Snail shRNA2 cells had decreased tumor size (Fig. 5E) after 26-day-injection. The size and weight measurements of tumors from PG Snail shRNA2 cells were also markedly decreased compared with control (Fig. 5F and G). Additionally, the quantification of human-specific Alu-sequences in the lungs was also significantly reduced following Snail knockdown (Fig. 5H). These results suggested that lower Snail expression reduced tumor growth and lung metastasis in lung cancer cells.

\section{Discussion}

Cell migration and invasion is the major cause of recurrence after surgery in lung cancer patients. A variety of studies have focused on exploring the mechanisms underlying tumor migration and invasion. Interestingly, during EMT, cancer cells acquire a fibroblastic phenotype induced by decreasing their cell-cell adhesions and become more motile (19). EMT is involved in the proliferation, invasion and stem cell features of cancer cells (20).

In the recent two decades, it has been appreciated that EMT is controlled by several transcriptional factors; however,
Snail, a member of the Snail family of zinc-finger transcription factors, is the key EMT regulator, directly repressing E-cadherin expression by binding to E-box elements in the E-cadherin promoter $(21,22)$. Snail had been correlated with a worse survival outcome in many malignancies (23-25). Additionally, Snail has been reported to regulate stem-like properties in thyroid and pancreatic cancer $(26,27)$. These findings suggest that Snail may serve as a putative molecular target for new therapeutics. Hence, clearly understanding the effects of Snail silencing on the biological behavior of lung cancer cells is important. In this context, we repressed Snail expression to investigate the role of Snail downregulation on the progression of lung cancer. We studied the correlation between Snail silencing and EMT processes in A549 cells, finding that Snail knockdown induced morphological changes and a less invasive phenotype compared with control cells. Inhibiting Snail enhanced expression of the epithelial marker E-cadherin and repressed the mesenchymal marker $\mathrm{N}$-cadherin, which has also been reported in melanoma (28), colon cancer (29), and hepatic carcinoma cells (30). These data explained the reduced migration following Snail interference.

To assess the effects of Snail knockdown on the biological behavior of lung cancer cells, we silenced Snail expression in lung cancer cell lines by isolating the Snail siRNA2 transfectant clones, and characterized the impact on growth and invasion in these lung cancer cells. Lung cancer cell lines with Snail knockdown were more invasive than the parental cell line in a previous study (31); however, in our study we found that Snail knockdown markedly decreased the proliferation and migration of A549 and PG cells. To uncover the mechanisms of these phenotypes, we analyzed genome-wide gene expression profiling studies. From these data, some cell cycle-associated genes were remarkably upregulated, such as P53AP1, CDK10, P21 and PAK1. However CDK2, CDK4, CDK6, Cyclin D and Cyclin E, most of the keys in G1/S phase checkpoint, were unaffected by Snail knockdown, as well as upon P27 (upstream of $C D K 2$ ), P15 and P19 (upstream of $C D K 4 / C D K 6)$. On the contrary, the major $\mathrm{G} 2 / \mathrm{M}$ phase checkpoints, such as $C d c 2$, $C y c l i n B$ and $C d c 25 A$, were markedly downregulated by Snail knockdown. From cell cycle analysis, we found that Snail knockdown activated the G2/M checkpoint protein $\mathrm{Cdc} 2$, but did not influence the G1 phase of the cell cycle. Moreover, the cell population was especially overrepresented from 12.8 to $34.9 \%$ in G2/M phase following Snail knockdown. Additionally, P21 and PCNA expression indicated that Snail knockdown could inhibit cell proliferation by enhancing P21 expression, which in turn, caused a decrease in PCNA expression. P21 can promote tumor cell proliferation and is dysregulated in many human cancers $(32,33)$, and also regulates PCNA expression by binding to the PCNA promoter, thereby inhibiting DNA replication and modulating DNA repair processes $(34,35)$. Furthermore, bioinformatics software analysis revealed that there are four potential consensus Snail-binding sites (CAGCTG at position $-1,061$ to $-1,055$ and -133 to -128 ; CACCTG at position $-1,068$ to $-1,063$ and -466 to -461 ) in the $\mathrm{P} 21$ promoter. These provide evidence of the relationship among Snail, P21, Cdc2 and PCNA. Thus, we hypothesize that a Snail/P21/Cdc2/PCNA signaling pathways exists and regulates lung cancer cell proliferation. 
Because Snail overexpression promoted lung cancer progression had been reported in vivo (36), we further examined the effects of silencing Snail on the tumorigenic behavior of A549 and PG cells in vivo to provide more persuasive evidence of tumor malignancy in knockdown cells. After injecting tumor cells into nude mice, we observed a decrease in tumor appearance and growth rate in the Snail knockdown group. Interestingly, the frequency of lung metastases was also reduced by Snail knockdown. This suggests that Snail knockdown not only interferes with proliferation, but also affects invasion and migration in vivo.

Altogether, this study showed that the inhibition of Snail expression can repress cell growth, invasion and metastasis of human lung cancer in vitro and in vivo. Furthermore, this study verified that Snail silencing impacted the malignant behavior of lung cancer cells by suppressing EMT processes and the cell cycle. Therefore, Snail could be a potential target for lung cancer treatment. Blocking Snail expression by siRNA could represent a new therapeutic strategy for lung cancer.

\section{Acknowledgements}

We would like to thank the FACS Core Facility of Peking University Cancer Hospital for performing FACS assays. This study was supported by the National Key Research and Development Program of China (2016 TFA 0500303), the National Natural Science Foundation of China (grant nos. 8133005, 81372594 and 81201964), the '863' Project (grant nos. 2014AA021606 and 2015AA020403), Beijing Natural Science Foundation (grant nos. 5122012 and 7132051).

\section{References}

1. Siegel RL, Miller KD and Jemal A: Cancer statistics, 2016. CA Cancer J Clin 66: 7-30, 2016.

2. Chen W, Zheng R, Baade PD, Zhang S, Zeng H, Bray F, Jemal A $\mathrm{Yu}$ XQ and He J: Cancer statistics in China, 2015. CA Cancer J Clin 66: 115-132, 2016.

3. Sher DJ, Fidler MJ, Liptay MJ and Koshy M: Comparative effectiveness of neoadjuvant chemoradiotherapy versus chemotherapy alone followed by surgery for patients with stage IIIA non-small cell lung cancer. Lung Cancer 88: 267-274, 2015

4. Moran C: Importance of molecular features of non-small cell lung cancer for choice of treatment. Am J Pathol 178: 1940-1948, 2011.

5. Thiery JP, Acloque H, Huang RY and Nieto MA: Epithelialmesenchymal transitions in development and disease. Cell 139: 871-890, 2009.

6. Thiery JP and Sleeman JP: Complex networks orchestrate epithelial-mesenchymal transitions. Nat Rev Mol Cell Biol 7: 131-142, 2006.

7. Iwatsuki M, Mimori K, Yokobori T, Ishi H, Beppu T, Nakamori S, Baba $\mathrm{H}$ and Mori M: Epithelial-mesenchymal transition in cancer development and its clinical significance. Cancer Sci 101: 293-299, 2010

8. Chen A, Beetham H, Black MA, Priya R, Telford BJ, Guest J, Wiggins GA, Godwin TD, Yap AS and Guilford PJ: E-cadherin loss alters cytoskeletal organization and adhesion in non-malignant breast cells but is insufficient to induce an epithelial-mesenchymal transition. BMC Cancer 14: 552, 2014.

9. Cano A, Pérez-Moreno MA, Rodrigo I, Locascio A, Blanco MJ, del Barrio MG, Portillo F and Nieto MA: The transcription factor snail controls epithelial-mesenchymal transitions by repressing E-cadherin expression. Nat Cell Biol 2: 76-83, 2000.

10. de Herreros AG, Peiró S, Nassour M and Savagner P: Snail family regulation and epithelial mesenchymal transitions in breast cancer progression. J Mammary Gland Biol Neoplasia 15: $135-147,2010$.
11. Come C, Magnino F, Bibeau F, De Santa Barbara P, Becker KF, Theillet $\mathrm{C}$ and Savagner P: Snail and slug play distinct roles during breast carcinoma progression. Clin Cancer Res 12: 5395-5402, 2006.

12. Kroepil F, Fluegen G, Vallböhmer D, Baldus SE, Dizdar L, Raffel AM, Hafner D, Stoecklein NH and Knoefel WT: Snaill expression in colorectal cancer and its correlation with clinical and pathological parameters. BMC Cancer 13: 145, 2013.

13. Cheon MG, Kim W, Choi M and Kim JE: AK-1, a specific SIRT2 inhibitor, induces cell cycle arrest by downregulating Snail in HCT116 human colon carcinoma cells. Cancer Lett 356B: 637-645, 2015.

14. Vega S, Morales AV, Ocaña OH, Valdés F, Fabregat I and Nieto MA: Snail blocks the cell cycle and confers resistance to cell death. Genes Dev 18: 1131-1143, 2004.

15. Lan L, Han H, Zuo H, Chen Z, Du Y, Zhao W, Gu J and Zhang Z: Upregulation of myosin Va by Snail is involved in cancer cell migration and metastasis. Int J Cancer 126: 53-64, 2010.

16. Xiao W, Chen X, Liu X, Luo L, Ye S and Liu Y: Trichostatin A, a histone deacetylase inhibitor, suppresses proliferation and epithelial-mesenchymal transition in retinal pigment epithelium cells. J Cell Mol Med 18: 646-655, 2014.

17. Su B, Zhao W, Shi B, Zhang Z, Yu X, Xie F, Guo Z, Zhang X, Liu J, Shen Q, et al: Let-7d suppresses growth, metastasis, and tumor macrophage infiltration in renal cell carcinoma by targeting COL3A1 and CCL7. Mol Cancer 13: 206, 2014.

18. Zijlstra A, Mellor R, Panzarella G, Aimes RT, Hooper JD, Marchenko ND and Quigley JP: A quantitative analysis of ratelimiting steps in the metastatic cascade using human-specific real-time polymerase chain reaction. Cancer Res 62: 7083-7092, 2002.

19. Yuen HF, Chan YK, Grills C, McCrudden CM, Gunasekharan V, Shi Z, Wong AS, Lappin TR, Chan KW, Fennell DA, et al: Polyomavirus enhancer activator 3 protein promotes breast cancer metastatic progression through Snail-induced epithelialmesenchymal transition. J Pathol 224: 78-89, 2011.

20. Talbot LJ, Bhattacharya SD and Kuo PC: Epithelial-mesenchymal transition, the tumor microenvironment, and metastatic behavior of epithelial malignancies. Int J Biochem Mol Biol 3: 117-136, 2012.

21. Shin NR, Jeong EH, Choi CI, Moon HJ, Kwon CH, Chu IS, Kim GH, Jeon TY, Kim DH, Lee JH, et al: Overexpression of Snail is associated with lymph node metastasis and poor prognosis in patients with gastric cancer. BMC Cancer 12: 521, 2012.

22. Kuo KT, Chou TY, Hsu HS, Chen WL and Wang LS: Prognostic significance of NBS1 and Snail expression in esophageal squamous cell carcinoma. Ann Surg Oncol 19 (Suppl 3): S549-S557, 2012

23. Hotz B, Arndt M, Dullat S, Bhargava S, Buhr HJ and Hotz HG: Epithelial to mesenchymal transition: Expression of the regulators snail, slug, and twist in pancreatic cancer. Clin Cancer Res 13: 4769-4776, 2007.

24. van Nes JG, de Kruijf EM, Putter H, Faratian D, Munro A, Campbell F, Smit VT, Liefers GJ, Kuppen PJ, van de Velde CJ, et al: Co-expression of SNAIL and TWIST determines prognosis in estrogen receptor-positive early breast cancer patients. Breast Cancer Res Treat 133: 49-59, 2012.

25. Kobayashi M, Huang CL, Sonobe M, Kikuchi R, Ishikawa M, Imamura N, Kitamura J, Iwakiri S, Itoi K, Yasumizu R, et al: Snail expression is associated with a poor prognosis in malignant pleural mesotheliomas. Ann Thorac Surg 95: 1181-1188, 2013.

26. Yasui K, Shimamura M, Mitsutake N and Nagayama Y: SNAIL induces epithelial-to-mesenchymal transition and cancer stem cell-like properties in aldehyde dehydroghenase-negative thyroid cancer cells. Thyroid 23: 989-996, 2013.

27. Zhou W, Lv R, Qi W, Wu D, Xu Y, Liu W, Mou Y and Wang L: Snail contributes to the maintenance of stem cell-like phenotype cells in human pancreatic cancer. PLoS One 9: e87409, 2014.

28. Liu S, Kumar SM, Martin JS, Yang R and Xu X: Snaill mediates hypoxia-induced melanoma progression. Am J Pathol 179: 3020-3031, 2011.

29. Zhang W, Wang J, Zou B, Sardet C, Li J, Lam CS, Ng L, Pang R, Hung IF, Tan VP, et al: Four and a half LIM protein 2 (FHL2) negatively regulates the transcription of E-cadherin through interaction with Snail1. Eur J Cancer 47: 121-130, 2011.

30. Zhang JP, Zeng C, Xu L, Gong J, Fang JH and Zhuang SM: MicroRNA-148a suppresses the epithelial-mesenchymal transition and metastasis of hepatoma cells by targeting Met/Snail signaling. Oncogene 33: 4069-4076, 2014. 
31. Merikallio H, Turpeenniemi-Hujanen T, Pääkkö P, Mäkitaro R, Riitta K, Salo S, Salo T, Harju T and Soini Y: Snail promotes an invasive phenotype in lung carcinoma. Respir Res 13: 104, 2012.

32. Liu H, Zhou L, Shi S, Wang Y, Ni X, Xiao F, Wang S, Li P and Ding K: Oligosaccharide G19 inhibits U-87 MG human glioma cells growth in vitro and in vivo by targeting epidermal growth factor (EGF) and activating p53/p21 signaling. Glycobiology 24: $748-765,2014$

33. Hu Z, Zhang D, Hao J, Tian K, Wang W, Lou H and Yuan H: Induction of DNA damage and p21-dependent senescence by Riccardin D is a novel mechanism contributing to its growth suppression in prostate cancer cells in vitro and in vivo. Cancer Chemother Pharmacol 73: 397-407, 2014.
34. Rousseau D, Cannella D, Boulaire J, Fitzgerald P, Fotedar A and Fotedar R: Growth inhibition by CDK-cyclin and PCNA binding domains of p21 occurs by distinct mechanisms and is regulated by ubiquitin-proteasome pathway. Oncogene 18: 3290-3302, 1999.

35. Zhao H, Ho PC, Lo YH, Espejo A, Bedford MT, Hung MC and Wang SC: Interaction of proliferation cell nuclear antigen (PCNA) with c-Abl in cell proliferation and response to DNA damages in breast cancer. PLoS One 7: e29416, 2012.

36. Yanagawa J, Walser TC, Zhu LX, Hong L, Fishbein MC, Mah V, Chia D, Goodglick L, Elashoff DA, Luo J, et al: Snail promotes CXCR2 ligand-dependent tumor progression in non-small cell lung carcinoma. Clin Cancer Res 15: 6820-6829, 2009. 